\title{
Gut micro biota, a key factor relating diet and inflammation with the progression of cognitive impairment in older people
}

\begin{abstract}
Developed societies are facing a remarkable increase of population's longevity and, consequently, of chronic diseases such as obesity, cardiovascular or neurodegenerative diseases. Age associated cognitive impairment (CI) (dementia) in its different forms represent a real social, economic and public health problem, and particularly the severe and highly prevalent Alzheimer disease (AD). Inflammation and altered metabolism of neurotransmitters and hormones are common in elderly suffering $\mathrm{CI}$ and $\mathrm{AD}$. Met genomics studies have demonstrated that in normal adult population, as well as in older people, health status and diet are the main factors affecting micro biota composition. Older persons frequently develop immune system degeneration leading to a low response to antigens and to a low profile persistent inflammation (inflammaging). Data from elderly populations and disease models show that systemic inflammation alters gut micro biota and also certain changes in the micro biota induce inflammatory secretions. In addition, there is a more than likely role of gut micro biota in the gut-brain signalling through the synthesis and processing of neurotransmitters. Hence, changes occurring in the gut micro biota of the elderly mostly due to diet, environment and inflammatory processes might be associated to brain function. The objective of this review would be to show that there are evidences that link gut micro biota and cognitive decline through inflammatory processes, although direct action on neurotransmitters may not be discarded. Research data shown here supports the view that inflammation linked to neurodegenerative diseases associated to ageing, may cause changes in the gut micro biota composition that, turn, would feed inflammation and aggravating the disease. This perverse cycle has been delayed to a certain extent through a healthy diet rich in complex carbohydrates, such as the Mediterranean diet.
\end{abstract}

Volume 6 Issue 2 - 2017

\author{
Gaspar Perez Martinez \\ Departamento de Biotecnología, Instituto de Agroquimíca y \\ Tecnología de Alimentos (C.S.I.C.),Valencia, Spain
}

Correspondence: Gaspar Pérez Martínez, Departamento de Biotecnología, Instituto de Agroquimíca y Tecnología de Alimentos (C.S.I.C.), Avenida Catedrático Agustín Escardino, 7, 46980 - Paterna,Valencia, Spain, Tel +34 963900022 (Ext. 202I), Fax+3496363630I, Email gaspar.perez@iata.csic.es

Received: October 01, 2016 | Published: February 15, 2017

Keywords: gut microbiota, ageing, diet, inflammation, cognitive impairmen

\begin{abstract}
Abbreviations: 5-HT, 5 hydroxy triptamine; $\mathrm{AD}$, alzheimer disease; $A \beta$, amyloid- $\beta$; $A \beta$ 1-42 and $A \beta$ 1-40, shorter peptides of $A \beta$ released during pathogenesis; ApoE, apolipoprotein E; CI, cognitive impairment; CMV, cytomegalovirus; CRP, c reactive protein; CSF, cerebrospinal fluid; CSN, central nervous system; DA, dopamine; EBV epstein-barr virus; GF, germ free; IBS, irritable bowel byndrome; IL-6; inter leukin 6; IL-8, inter Leukin8; MAMPS, microbe associated molecular patter NA, noradrenalin PET, positron emission tomography; PPA, precursor protein amyloi; p-tau, phosphorylated tau protein; SCFA, short chain fatty acid; SLRP, soluble low density lipoprotein receptor-related protein-1; SPF, specific pathogen free; sRAGE, soluble receptor for advanced glycation end products; $\mathrm{TNF}-\alpha$, tumor necrosis factor alpha
\end{abstract}

\section{Introduction}

The aim of this first part is providing solid grounds to propose links between gut microbiota and cognitive decline through inflammatory processes, although direct action on neurotransmitters may also have an incidence. Hence, it will be needed to update the state of the art on $\mathrm{CI}$ and $\mathrm{AD}$, and their links with inflammatory processes will be revealed through the study of molecular markers. Finally, the relationship of gut micro biota with brain function and changes during ageing will be reviewed.

\section{Cognitive impairment and alzheimer disease}

Life expectancy has greatly improved in the last century, generating unprecedented social and public health problems, due to the great increase of the older population. In fact, different forecasts suggest that $50 \%$ of today's young people will reach 90years and, if present proportions persist, between $40-60 \%$ of them will suffer some sort of cognitive impairment or AD after that age (90years). Hence, if we are unable to find a solution, in two to three decades there will be more dements than ever before in human history. ${ }^{1,2}$ Unfortunately, all studies revealed that age is the main risk factor for $\mathrm{CI}$ or $\mathrm{AD}$ and it duplicates every 10years, therefore, our "oldest old" - that is older than 90years- are those at greater risk. Different works have tried to determine other risk factors and although a significant correlation has been found between longevity and the intake of vitamins, antioxidants, physical activity, caffeine, alcohol or body mass index, it was not possible to find any relationship with the development of neurodegenerative diseases. Among all factors studied that may influence the development of $\mathrm{CI}$ or $\mathrm{AD}$, only hypertension has been clearly determined to reduce and hypotension to increase the risk to develop dementia. The causes are yet undetermined, but it has been suggested that brain capillaries develop rigid walls with age and contorted paths require greater tension for the adequate perfusion of nutrients and oxygen..$^{3-5}$ The presence of amyloid- $\beta(\mathrm{A} \beta)$ protein 
is a definite risk factor, as $60 \%$ of old persons with dementia show presence of $\mathrm{A} \beta$ after positron emission tomography (PET) scan, and $40 \%$ of healthy volunteers of similar age $(>90)$ showed the presence of $A \beta$. However, this healthy group positive for $A \beta$ lost their cognitive faculties faster than the healthy group with no $A \beta$ signal. ${ }^{6} A \beta$ plates have an important role in $\mathrm{AD}$ pathogenesis, but the severity of the cognitive impairment holds greater correlation with the burden of neocortical neurofibrillary tangles than with the presence of $\mathrm{A} \beta$ plates. $^{7}$ In elderly of very advanced age (more than 90-95years), the pathology of $\mathrm{AD}$ depends rather on genetic factors than on age itself due to the appearance of the precursor protein amyloid- $\beta$ (PPA).Nowadays, different causes of dementia can be histological differentiated among them, of course, $\mathrm{AD}$ and hippocampus sclerosis. The main neuropath logical features of $\mathrm{AD}$ are the loss of neurons and synopsis, the formation of plates (of $A \beta$ protein) and neurofibrillary tangles (aggregates of tau protein associated to microtubules) in the cortical and limbic regions of the brain, and until present, the most efficient diagnostic of $\mathrm{AD}$ is obtained through lumbar puncture for the detection of $A \beta 1-42$ and $A \beta 1-40$, total tau protein or phosphorylated tau (p-tau) ${ }^{8,9} \mathrm{CI}$ and $\mathrm{AD}$ can be classified as diseases of inflammatory nature due to the high concentrations of inflammatory markers detected (see below); prolonged anti-inflammatory treatments reduce the risk of their development. Although the causal relationship between inflammation and $\mathrm{CI}$ or $\mathrm{AD}$ has not been disclosed, but some molecular components of inflammatory events promote pathological processes that lead to $\mathrm{AD}$, while others have the opposite effect. This apparently depends on the way they affect different types of cells in the brain of different individuals. ${ }^{10}$

\section{Investigation and diagnostic of cognitive impairment} and alzheimer disease through molecular methods

The clinical diagnostic of AD is mainly based on neuropsychological symptoms and until recently this disease required the post mortem confirmation of the presence of $A \beta$ plates and fibrillary tangles in the cortex for $100 \%$ precise diagnostic. ${ }^{11}$ Therefore, in the last years a great effort has been devoted to determine molecular markers for robust, and if possible, early diagnostics of AD. The protein analysis of cerebrospinal fluid (CSF) ( $\beta$ A 1-42 (INNOTEST ${ }^{\circledR}$ ), ApoE, tau and phosphorylated tau is now considered the most precise method for the diagnostic of AD. ${ }^{12-15}$ Attempts to develop less invasive methods were published based on the detection of multiple plasma molecular markers (between 18 and 89) that showed a precision of $70 \%$ for early detection of $\mathrm{CI}$ and $\mathrm{AD} .^{16,17}$ Later studies developed methods for the analysis of individual markers in plasma, such as the presence of the soluble form of the receptor for advanced glycation end products (sRAGE) and the soluble low density lipoprotein receptorrelated protein-1 (sLRP). ${ }^{18}$ Metabolites of different fluids have been characterized through metabolomics in $\mathrm{AD}$ transgenic mouse models and humans with $\mathrm{AD}$ and $\mathrm{CI}$. In general terms, detected products in plasma and CSF followed parallel patterns and likely differences were found between normal elderly people and those affected by AD or CI (up to $40-60 \%$ of total compounds). Differential patterns were mainly found in canonical pathways related to the energy metabolism and mitochondrial function, lipid biosynthesis and transport, and metabolism of neurotransmitters and hormones. Remarkably, some of these signatures and profiles can be explained by the metabolism of the gut microbiota, as will be reviewed below. ${ }^{19-23}$ The detection of individual compounds can be used as a fast diagnostic method. For example, the presence of cortisol in saliva could be shown in a number of works that holds direct relationship with stress, ${ }^{24}$ determined through ELISA techniques. ${ }^{25}$ However, cortisol determination can also be related to neurodegenerative processes, especially $\mathrm{CI}$ and $\mathrm{AD}$, as marker of the hypothalamus and the adrenocortical gland activity $^{26}$ and it can be determined as a typical CSF metabolite in AD. ${ }^{27}$ Since 2005 gene expression studies (transcriptomics) tried to discriminate differences between healthy volunteers and $\mathrm{CI}$ and $\mathrm{AD}$ patients using DNA microarrays on nervous tissue and CSF samples. ${ }^{28}$ The amount of information obtained was precise and abundant, but those studies underestimated posttranscriptional events, offering only a partial view of such biological problems. On the other hand, the transcriptomic analysis of blood cells may offer a more general view on the status of the organism. A very complete blood study by Lunnon $\mathrm{K}$ et al. ${ }^{29}$ described differential expression of more than 2,900 probes between controls and patients with $\mathrm{CI}$ and $\mathrm{AD}$. The most significant group (77) had lower level of expression in the subjects affected by $\mathrm{CI}$ and $\mathrm{AD}$, from which authors underpinned genes mainly related to the respiratory chain of mitochondria. Of note, the only group of genes significantly over expressed in the disease cases were related with inflammatory immune response, particularly pathways related to endothelial leukocyte migration, oxidative phosphorylation and ribosomal synthesis. ${ }^{29}$

Finally, newly developed imaging techniques need to be mentioned. PET and magnetic resonance imaging (MRI) are noninvasive methods with great in vivo resolution that have successfully been applied to humans. PET with 2-[18F] fluoro-2-deoxy-D-glucose (FDG) has been globally used for more than 20years. This probe detects general levels of metabolic activity, and images obtained have enough resolution for some expert professionals to determine if patients are suffering CI or AD. ${ }^{30}$ In the past years, radiolabelled probes applied are able to directly detect $A \beta$ with great efficacy, for example, results using [11C]-Pittsburg compound-B (PIB) rendered a robust diagnostic of the fibrillary deposition of the amyloid protein in the neocortex on 238 volunteers of a multicenter study. ${ }^{31}$ Also the ratio of $A \beta$ 1-42/ $A \beta$ 1-40 using high precision mass spectroscopy were definitely associated with the presence of cortical $A \beta$ fibrils. ${ }^{32}$

\section{Gut microbiota and the gut-brain axis}

Humans have coevolved during millions ofyears with a variety of microbial species in such a way that we need a balanced gut microflora (microbiota) for food digestion, pathogen inhibition, preservation of the gut mucosa integrity, so that from the moment of birth, we depend on the gut microbiota to achieve a proper maturation of the immune system. ${ }^{33,34}$ Changes in the composition of the microbiota exert an effect on the host physiology, contributing to the development of diseases that involve inflammatory disorders, type II diabetes, obesity, metabolic syndrome for reviews $\mathrm{se}^{35-38}$ and liver functions. ${ }^{39}$ The axis Brain-Intestine has long been known to affect intestinal functions under stress or anxiety conditions (up-down signal). Here we will focus on the "down-up" signal and will show some experimental examples that highlight such Gut-Brain communication mechanism. The fact that gut micro biota can transcend the digestive and immune functions is still confronted by the skepticism of some clinicians, but there are sound scientific evidences of the existence of signaling pathways between gut micro biota and the brain. Four transport paths have been proposed for signals from Gut (micro biota) to Brain: the vagus nerve and spinal efferent systems; mediators of the immune system, such as cytokines; enteric hormones; and finally, signaling molecules produced by the gut micro biota. ${ }^{40}$ Some examples are following that will shed light on these mechanisms. It has been shown that germ free (GF) mice displayed greater motor activity and less anxiety than mice colonized with normal micro biota (SPF). This 
difference can be attributed to an increase in noradrenalin (NA), dopamine (DA) and 5-hydroxytriptamine (5-HT) turnover in the striatum of GF mice, known monoamine neurotransmitters associated to anxiety-like behavior, and to changes in expression of genes that participate in the synaptic plasticity. ${ }^{39}$ In a different study, GF male mice showed a significant increase in hippocampus concentration of 5-HT (serotonin), if compared to conventionally colonized animals. In fact, the concentration of tryptophan, precursor of serotonin, was higher in plasma of GF mice, suggesting that gut microbiota may influence serotonergic neurotransmission of the central nervous system (CNS) via humoral communication path. ${ }^{41}$ Different works could demonstrate that the vagus nerve serves as an important neurotransmitter of the changes occurring in the gut microbiota These studies described assays in which the intake of probiotc strains, Lactobacillus rhamnosus and Bifidobacterium longum, did not reduce inflammatory markers, however, they managed to normalize the anxiety related behavior caused by colitis, a process mediated by the vagus nerve that is possibly activated at the level of the enteric nervous system. ${ }^{42}$ Particularly, L. rhamnosus (JB-1) induced gene expression changes of the receptor of $\gamma$-aminobutiric acid (GABA) in different regions of the brain, as well as a reduction of the levels of corticosterone and the anxiety/depression related behavior. Those behavioral and neuro chemical effects were not present in vagotomized mice. ${ }^{43}$ Catecholamines (CA) can also be involved in the Gut-Brain signaling regulated by gut bacteria. Much lower levels of dopamine and norepinephrine have been found in the intestinal lumen of GF mice than in conventional SFP mice, further, CA found in SPF mice were biologically active, while those in GF mice were conjugated or inactive. ${ }^{44}$ Manipulation of the micro biota in rodents gives rise to changes in the regulation of anxiety, mood, cognition and pain, that led to suggest that proper modification of the gut microbiota could be an optional strategy in the development of new therapies in complex CNS disorders. ${ }^{45}$ In this line, some studies related changes in the gut micro biota with autism, anxiety and depression also in humans, however, the signaling channels are still under research. ${ }^{46}$ It could be demonstrated that chronic intestinal infection in animal models is related to an anxiety and depression related behavior, effects that are associated to changes in the patterns of proinflammatory cytokines and metabolic pathways of kinurenine/tryptophan. ${ }^{47}$ In humans, the irritable bowel syndrome (IBS) is correlated to intestinal inflammation and a strong deviation of the normal gut microbial pattern (dysbiosis). Since gut inflammation is the basis of systemic inflammation, endotoxemia and neuro inflammation, this would explain why a high proportion of IBS patients (also consuming alcohol, tobacco and high fat diets), have a high risk to suffer respiratory, neuromuscular, psychological, sleep or neurological disorders, stressing the effect of the communication paths between dysbiosis, gut and the CNS. ${ }^{48}$ In a mouse model of the ME7 prion disease, systemic inflammation induced acute cognitive deficiencies. ${ }^{49}$ Regarding the incidence of the general inflammatory condition on dementia in humans, a recent meta-analysis demonstrated that high levels of peripheral inflammatory markers are related to a slight increase in the risk of dementia in the broad sense, not to AD. ${ }^{50}$

\section{Microbiota and the immune system in the elderly}

Ageing brings along deregulation of the immune response due to a life-long hyper stimulation of the immune system (immuno senescence). At advanced age, there is a lower response to antigens due to thyme involution, with a reduction of total $\mathrm{T}$ and $\mathrm{B}$ cells, and naïve $\mathrm{T}$ cells (CD95-) needed for the production of new immunoglobulins (Ig) against pathogens. As consequence of age, there is also an accumulation of effector $\mathrm{T}$ cells $(\mathrm{CD} 8+)$ that produce pro-inflammatory cytokines, an increase of Natural Killer (NK) cells and a progressive activation of macrophages, all this is displayed with constant low grade inflammatory signaling (inflammaging). This inflammatory stage in elderly people has been related to different age related diseases, from diabetes to cardiovascular or neurodegenerative diseases for review see. ${ }^{51}$ Certain viral infections, such as Epstein-Barr Virus (EBV) and cytomegalovirus (CMV). ${ }^{52}$ Significantly participate in the development of the chronic pro-inflammatory state in elderly. This inflammatory scenario sensitizes elderly to microbe associated molecular patterns (MAMPS) that originate an inflammatory loop that may explain why changes in micro biota composition are related with the progression of diseases and frailty in older population, although at present we cannot explain what occurs first. ${ }^{53}$ Thus, the maintenance of a stable and "healthy" micro biota could prevent or delay the inflammatory state associated to age (inflammaging). Certain bacterial groups, such as Faecal bacterium, lactobacilli and bifido bacteria can modulate the pro-inflammatory response..$^{54-57}$ In fact, the presence of strains of the genus Bifidobacterium in the gut micro biota of elderly is negatively correlated with high levels of the pro-inflammatory cytokine TNF- $\alpha$ and with the interleukine 10 (IL-10).$^{58}$ On the other hand, the increase of Enterobacteriaceae and other Proteobacteria is related to the reduction of short chain fatty acids (SCFA) in feces, lower secretion of mucins, a greater stimulation of bacterial endotoxins, as well as the reduced permeability of the intestinal barrier, favoring the severity of the immune response and reducing the tolerance to otherwise harmless commensal bacteria, which under those conditions are turned into a strong pro-inflammatory stimulus. ${ }^{59,60}$ Micro biota changes with age and it was proposed that older people have a higher proportion of Bacteroidetes vs. Firmicutes, and low presence of bifidobacteria. ${ }^{61}$ However, recent studies could not find significant differences between micro biota in healthy adults and elderly of more than 65years. Nevertheless in older persons, there is a clear relationship between the health status, diet and age related decline, particularly parameters of frailty, co-morbidity, nutritional status, inflammatory markers and fecal water metabolites. Inflammatory markers (TNF- $\alpha$, IL-6, IL- 8 and the C reactive protein (CRP)) had higher levels in institutionalized subjects than in community dwelling elderly. ${ }^{62}$ This relationship between the immune system, microbiota and age becomes relevant in AD patients. The immune system of old persons susceptible to AD could be weaker, as deduced from the significant relationship between the evolution towards mild cognitive impairment and $\mathrm{AD}$ and the presence of antibodies against bacteria typically associated to periodontal infections, such as Fusarium nucleatum and Prevotella intermediate. ${ }^{63}$

\section{Discussion}

During the lastyears, human studies on the composition of the gut microbiota have generated massive amounts of data in different individuals and conditions. One of the main observations was that significant relationships could be established between "core" bacterial populations in the gut associated to different diets. Initially, three enterotypes were described after the dominant bacterial groups, they were the Bactericides enterotype, associated to diets rich in protein, simple sugars and animal fat and, Prevotella and Ruminococcus enterotypes related to high fiber intake. ${ }^{64}$ Subsequent publications simplified this classification to Bacteroides and Prevotella enterotypes. ${ }^{65}$ The Prevotella entero type was not only bound to complex carbohydrate consumption but resulted in a more efficient utilization of fiber and release of beneficial SCFAs in individuals carrying this enterotype. ${ }^{66}$ Similar conclusion was drawn by a study (cited above) that constitutes a landmark of the gut micro biota research in elderly. ${ }^{62}$ 
These authors analyzed micro biota of 178 volunteers $\geq 65$ years old and obtained the covariance with other factors, like community dwelling vs. long stay in institutions, clinical indicators of frailty, inflammation and dietary profiles. This study clearly determined that a long stay in institutions was bound to different bacterial groups and to inflammatory markers (TNF- $\alpha$, IL- 6 and IL- 8 and CRP), but also found that individuals in institutions followed a diet richer in animal fat and with less fiber. These volunteers had a less diverse micro biota, a lower functional independence, depression, and mini-mental cognitive test scores. A gradient was observed as individuals changed from community dwelling to long stay in institutions, following a decrease of SCFAs in feces and bacteria from the genera Prevotella and Ruminococcus. This work suggested a clear connection between gut micro biota/diet and mental status in older persons. The Mediterranean diet has a direct impact on the gut micro biota can be established through its high content in vegetables and complex carbohydrates that favor the proliferation of Prevotella ${ }^{67}$ Furthermore, recent meta-analysis reported that a better adherence to Mediterranean diet can delay cognitive decline.$^{68}$ And even ApoE $\varepsilon 4$ allele carriers had a better performance in executive function after 36 months. ${ }^{69}$ Both studies suggesting a fundamental role of the gut microbiota. But how are intestinal bacteria connected to brain function? Different studies mentioned above showed that changes in the gut microbiota lead to altered expression of anxiety and synaptic plasticity genes. ${ }^{39}$ And changes in the behavior (anxiety and social interaction) of mice. ${ }^{70}$ However, due to intrinsic difficulties of the mouse models, a clear connection could not be established with cognitive faculties but, in humans, depression in inflammatory diseases, like IBS, have been related to cognitive decline. ${ }^{48}$ Then, inflammation can be the link. $\mathrm{AD}$ is considered disease where inflammation does not exclusively affect the CNS, it also implies systemic dysfunction of metabolic, inflammatory, and biochemical pathways. Certainly, inflammaging was described as a low-grade, controlled, asymptomatic, chronic, systemic, inflammatory state, affecting older people has been related to many age related diseases. ${ }^{71}$ And particularly to frailty and faster cognitive decline in the elderly. ${ }^{72-74}$

\section{Conclusion}

This is a multidisciplinary topic that is developing very fast in the last few years, as ageing health complications and the need for empowerment of older citizens constitute institutional priorities in modern society. Other comprehensive reviews have tried to condense the numerous interacting factors and very complex causalities of cognitive impairment including frailty, depression, inflammation or oxidative stress among others. ${ }^{73}$ This review has a different aim, to summarize scientific reports in a simple scheme that could connect four main factors: age, gut micro biota, diet and inflammation with cognitive decline (Figure 1). Age prompted inflammaging, results in a chronic, systemic inflammation that has a direct impact on the composition of the gut micro biota, but also causes the general health decline, including frailty, depression and accelerates AD and dementia. Diet and possible dietary interventions (pro biotics and pre biotics) may help to manage deviations of the gut micro biota. Particularly, the Mediterranean diet has a positive impact on the micro biota, increasing the populations of Prevotella and the production of beneficial SCFAs by gut bacteria. Although out of this context, this diet also provides Omega 3 and Omega 6 fatty acids that improve cardiovascular disease, a health problem with a known negative impact on cognitive performance.

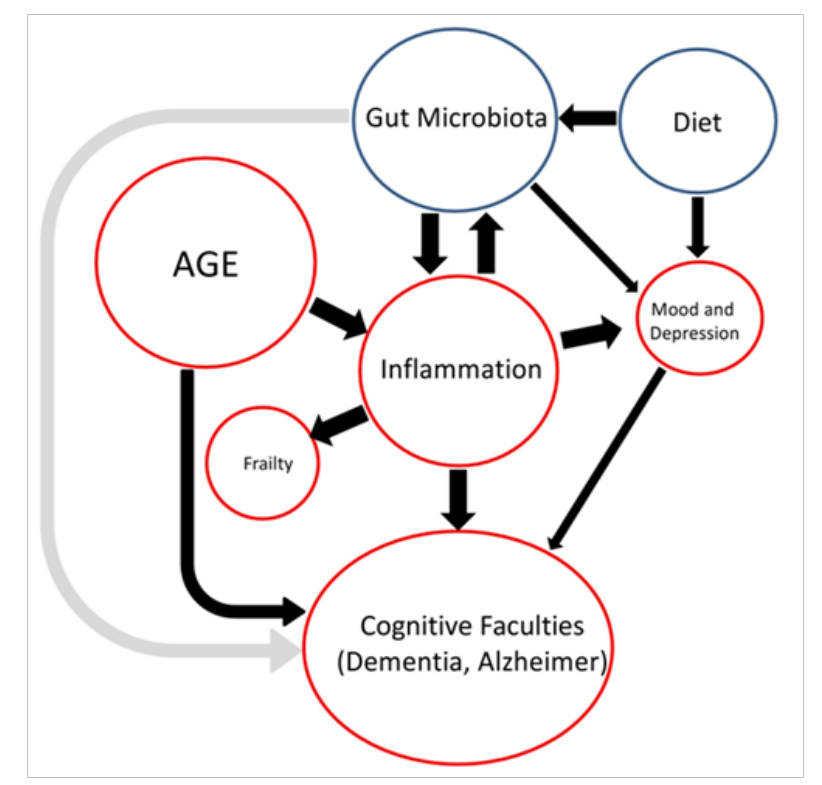

Figure I Scheme representing demonstrated (black arrows) and modelled relationships (grey arrow) occurring during ageing between Gut Micro biota, Diet, Mood and Depression, Inflammation and Cognitive Faculties, where inflammatory processes play a central role. This is described in detail in the Discussion and Conclusions.

\section{Acknowledgments}

Author wishes to thank Spanish Ministerio de Economia y Competitividad projects AGL2013-47420-R and AGL2015-70487-P for the financial support.

\section{Conflict of interest}

Author declares that there is no conflict of interest.

\section{References}

1. Bullain SS, Corrada MM. Dementia in the oldest old. Continuum (Minneap Minn). 2013;19(2):457-469.

2. Christensen K, Doblhammer G, Rau R, et al. Ageing populations: the challenges ahead. The Lancet. 2009;374(9696):1196-1208.

3. Morris MC, Scherr PA, Hebert LE, et al. Association of incident alzheimer disease and blood pressure measured from 13 years before to 2 years after diagnosis in a large community study. Archives of Neurology. 2001;58(10):1640-1646

4. Nilsson SE, Read S, Berg S, et al. Low systolic blood pressure is associated with impaired cognitive function in the oldest old: longitudinal observations in a population-based sample 80years and older. Aging Clin Exp Res. 2007;19(1):41-47.

5. Ruitenberg A, den Heijer T, Bakker SL, et al. Cerebral hypoperfusion and clinical onset of dementia: the Rotterdam Study. Ann Neurol. 2005;57(6):789-794.

6. Kawas CH, Greenia DE, Bullain SS, et al. Amyloid imaging and cognitive decline in nondemented oldest-old: The 90+ Study. Alzheimer's \& dementia: the journal of the Alzheimer's Association. 2013;9(2):199-203.

7. Nelson PT, Alafuzoff I, Bigio EH, et al. Correlation of Alzheimer disease neuropathologic changes with cognitive status: a review of the literature. J Neuropathol Exp Neurol. 2012;71(5):362-381. 
8. Krut JJ, Zetterberg H, Blennow K, et al. Cerebrospinal fluid Alzheimer's biomarker profiles in CNS infections. Journal of Neurology. 2013;260(2):620-626

9. Nelson PT, Schmitt FA, Lin Y, et al. Hippocampal sclerosis in advanced age: clinical and pathological features. Brain. 2011;134(5):1506-1518.

10. Wyss-Coray T, Rogers J. Inflammation in Alzheimer Disease-A Brief Review of the Basic Science and Clinical Literature. Cold Spring Harbor Perspectives in Medicine. 2012;2(10):a006346.

11. Sabbagh MN, Cooper K, DeLange J, et al. Functional, global and cognitive decline correlates to accumulation of Alzheimer's pathology in MCI and AD. Curr Alzheimer Res. 2010;7(4):280-286.

12. Blennow K, Zetterberg H. The Application of Cerebrospinal Fluid Biomarkers in Early Diagnosis of Alzheimer Disease. Medical Clinics of North America. 2013;97(3):369-376.

13. Monge Argiles A, Blanco Canto MA, Leiva Salinas C, et al. A comparison of early diagnostic utility of Alzheimer disease biomarkers in brain brain magnetic resonance and cerebrospinal fluid. Neurologia. 2013;29(70):397-401

14. Rosen C. Zetterberg H. Cerebrospinal fluid biomarkers for pathological processes in Alzheimer's disease. Curr Opin Psychiatry. 2013;26(3):276-282.

15. Wang C, Yu JT, Wang HF, et al. Meta-Analysis of Peripheral Blood Apolipoprotein E Levels in Alzheimer's Disease. PLoS ONE. 2014;9(2):e89041.

16. Ray S, Britschgi M, Herbert C, et al. Classification and prediction of clinical Alzheimer's diagnosis based on plasma signaling proteins. Nat Med. 2007;13:1359-1362.

17. Soares HD, Chen Y, Sabbagh M, et al. Identifying Early Markers of Alzheimer's Disease using Quantitative Multiplex Proteomic Immunoassay Panels. Annals of the New York Academy of Sciences. 2009;1180:56-67.

18. Liang F, Jia J, Wang S, et al. Decreased plasma levels of soluble low density lipoprotein receptor-related protein-1 (sLRP) and the soluble form of the receptor for advanced glycation end products (sRAGE) in the clinical diagnosis of Alzheimer's disease. Journal of Clinical Neuroscience. 2013;20(3):357-361.

19. Greenberg N, Grassano A, Thambisetty M, et al. A proposed metabolic strategy for monitoring disease progression in Alzheimer's disease. Electrophoresis. 2009;30(7):1235-1239.

20. Motsinger-Reif A, Zhu H, Kling M, et al. Comparing metabolomic and pathologic biomarkers alone and in combination for discriminating Alzheimer's disease from normal cognitive aging. Acta Neuropathol Commun. 2013;1:28.

21. Orešič M, Hyötyläinen T, Herukka SK, et al. Metabolome in progression to Alzheimer's disease. Transl Psychiatry. 2011;1:e57.

22. Trushina E, Mielke MM. Recent advances in the application of metabolomics to Alzheimer's Disease. Biochimica et Biophysica Acta (BBA) - Molecular Basis of Disease. 2013;1842(8):1232-1239.

23. Zhu H, Luo M. Chemical structure informing statistical hypothesis testing in metabolomics. Bioinformatics. 2014;30(4):514-522.

24. Hellhammer DH, Wüst S, Kudielka BM. Salivary cortisol as a biomarker in stress research. Psychoneuroendocrinology. 2009;34(2):163-171.

25. Inder WJ, Dimeski G, Russell A, Measurement of salivary cortisol in 2012 - laboratory techniques and clinical indications. Clin Endocrinol (Oxf). 2012;77(5):645-651.
26. Meyer JS, Novak MA. Minireview: Hair Cortisol: A Novel Biomarker of Hypothalamic-Pituitary-Adrenocortical Activity. Endocrinology. 2012;153(9):4120-4127.

27. Czech C, Berndt P, Busch K, et al. Metabolite Profiling of Alzheimer's Disease Cerebrospinal Fluid. PLoS One. 2012;7(2):e31501.

28. Ho L, Sharma N, Blackman L, et al. From proteomics to biomarker discovery in Alzheimer's disease. Brain Research Reviews. 2005;48(2):360-369.

29. Lunnon K, Ibrahim Z, Proitsi P, et al. Mitochondrial Dysfunction and Immune Activation are Detectable in Early Alzheimer's Disease Blood. Journal of Alzheimer's Disease. 2012;3093:685-710.

30. Mosconi L, Berti V, Glodzik L, et al. Pre-Clinical Detection of Alzheimer's Disease Using FDG-PET, with or without Amyloid Imaging. Journal of Alzheimer's Disease. 2010;20(3):843-854.

31. Nordberg A, Carter SF, Rinne J, et al. A European multicentre PET study of fibrillar amyloid in Alzheimer's disease. European Journal of Nuclear Medicine and Molecular Imaging. 2013;40(1):104-114.

32. Pannee J, Portelius E, Minthon L, et al. Reference measurement procedure for CSF $A \beta 1-42$ and the CSF A $\beta 1-42 / A \beta 1-40$ ratio - a cross-validation study against Amyloid PET. J Neurochem. 2016;139(4):651-658.

33. Collado MC, Cernada M, Baüerl C, et al. Microbial ecology and host-microbiota interactions during early life stages. Gut Microbes. 2012;3(4):352-365

34. Zeissig S, Blumberg RS. Life at the beginning: perturbation of the microbiota by antibiotics in early life and its role in health and disease. Nat Immunol. 2014;15(4):307-310.

35. Blaut M, Klaus S. Intestinal microbiota and obesity. Handb Exp Pharmacol. 2012;(209):251-273.

36. Gruber L, Lichti P, Rath E, et al. Nutrigenomics and nutrigenetics in inflammatory bowel diseases. J Clin Gastroenterol. 2012;46(9):735-747.

37. Kelly D, Mulder IE. Microbiome and immunological interactions. Nutr Rev. 2012;70(Suppl 1):1753-4887.

38. Tremaroli V, Bäckhed F. Functional interactions between the gut microbiota and host metabolism. Nature. 2012;489(7415):242-249.

39. Diaz Heijtz R, Wang S, Anuar F, et al. Normal gut microbiota modulates brain development and behavior. Proceedings of the National Academy of Sciences. 2011;108(7):3047-3052.

40. Holzer P, Farzi A. Neuropeptides and the Microbiota-Gut-Brain Axis Adv Exp Med Biol. 2014;817:195-219.

41. Clarke G, Grenham S, Scully P, et al. The microbiome-gut-brain axis during early life regulates the hippocampal serotonergic system in a sexdependent manner. Mol Psychiatry. 2013;18(6):666-673.

42. Bercik P, Park AJ, Sinclair D, et al. The anxiolytic effect of Bifidobacterium longum NCC3001 involves vagal pathways for gut-brain communication. Neurogastroenterology \& Motility. 2011;23(12):1132-1139.

43. Bravo JA, ForsytheP, Chew MV, et al. Ingestion of Lactobacillus strain regulates emotional behavior and central GABA receptor expression in a mouse via the vagus nerve. Proceedings of the National Academy of Sciences. 2011;108(38):16050-16055.

44. Asano Y, Hiramoto T, Nishino R, et al. Critical role of gut microbiota in the production of biologically active, free catecholamines in the gut lumen of mice. Am J Physiol Gastrointest Liver Physiol. 2012;303(1):1288-1295 
45. Cryan JF, Dinan TG. Mind altering microorganisms: the impact of the gut microbiota on brain and behaviour. Nat Rev Neurosci. 2012;13(10):701-712

46. Mayer EA. Gut feelings: the emerging biology of gut-brain communication. Nat Rev Neurosci. 2011;12(8):453-466.

47. Bercik P, Collins SM. The effects of inflammation, infection and antibiotics on the microbiota-gut-brain axis. Adv Exp Med Biol. 2014;817:279-289.

48. Daulatzai M. Chronic Functional Bowel Syndrome Enhances Gut-Brain Axis Dysfunction, Neuroinflammation, Cognitive Impairment, and Vulnerability to Dementia. Neurochem Res. 2014;39(4):624-644.

49. Murray C, Sanderson DJ, Barkus C, et al. Systemic inflammation induces acute working memory deficits in the primed brain: relevance for delirium. Neurobiology of Aging. 2012;33(3):603-616.

50. Koyama A, O'Brien J, Weuve J, et al. The Role of Peripheral Inflammatory Markers in Dementia and Alzheimer's Disease: A MetaAnalysis. The Journals of Gerontology Series A: Biological Sciences and Medical Sciences. 2013;68(4):433-440.

51. Pérez Martínez G, Bäuerl C, Collado MC. Understanding gut microbiota in elderly's health will enable intervention through probiotics. Beneficial Microbes. 2014;5(3):235-246.

52. Sansoni P, Vescovini R, Fagnoni F, et al. The immune system in extreme longevity. Experimental Gerontology. 2008;43(2):61-65.

53. Guigoz Y, Doré J, Schiffrin EJ. The inflammatory status of old age can be nurtured from the intestinal environment. Current Opinion on Clinical Nutrition and Metabolic Care. 2008;11(1):13-20.

54. Bäuerl C, Llopis M, Antolín M, et al. Lactobacillus paracasei and Lactobacillus plantarum strains downregulate proinflammatory genes in an ex vivo system of cultured human colonic mucosa. Genes Nutr. 2013;8(2):165-180.

55. Heuvelin $\mathrm{E}$, Lebreton $\mathrm{C}$, Grangette $\mathrm{C}$, et al. Mechanisms Involved in Alleviation of Intestinal Inflammation by Bifidobacterium Breve Soluble Factors. PLoS ONE. 2009;4(4):e5184.

56. Sokol H, Pigneur B, Watterlot L, et al. Faecalibacterium prausnitzii is an anti-inflammatory commensal bacterium identified by gut microbiota analysis of Crohn disease patients. Proceedings of the National Academy of Sciences. 2008;105(43):16731-16736

57. Van Baarlen P, Troost F, van der Meer C, et al. Human mucosal in vivo transcriptome responses to three lactobacilli indicate how probiotics may modulate human cellular pathways. PNAS. 2010;108(Suppl 1):4562-4569

58. Ouwehand AC, Bergsma N, Parhiala R, et al. Bifidobacterium microbiota and parameters of immune function in elderly subjects. FEMS Immunology and Medical Microbiology. 2008;53(1):18-25.

59. Biagi E, Nylund L, Candela M, et al. Through Ageing, and Beyond: Gut Microbiota and Inflammatory Status in Seniors and Centenarians. PLoS One. 2010;5(5):e10667.
60. Schiffrin EJ, Morley JE, Donnet-Hughes A, et al. The inflammatory status of the elderly: The intestinal contribution. Mutation Research Fundamental and Molecular Mechanisms of Mutagenesis. 2010;690(1-2):50-56

61. Mariat D, Firmesse O, Levenez F, et al. The Firmicutes/Bacteroidetes ratio of the human microbiota changes with age. BMC Microbiology. 2009;9:123.

62. Claesson MJ, Jeffery IB, Conde S, et al. Gut microbiota composition correlates with diet and health in the elderly. Nature. 2012;488(7410):178-184.

63. Sparks Stein P, Steffen MJ, Smith C, et al. Serum antibodies to periodontal pathogens are a risk factor for Alzheimer's disease. Alzheimer's \& Dementia. 2012;8(3):196-203.

64. Arumugam M, Raes J, Pelletier E, et al. Enterotypes of the human gut microbiome. Nature. 2011;473(7346):174-180.

65. Wu GD, Chen J, Hoffmann C, et al. Linking Long-Term Dietary Patterns with Gut Microbial Enterotypes. Science. 2011;334(6052):105-108.

66. Chen T, Long W, Zhang, C, et al. Fiber utilizing capacity varies with Prevotella versus Bacteroides enterotypes. The FASEB Journal. 2016;30(2):683-682.

67. De Filippis F, Pellegrini N, Vannini L, et al. High-level adherence to a Mediterranean diet beneficially impacts the gut microbiota and associated metabolome. Gut. 2016;65(11):1812-1821.

68. Van de Rest O, Berendsen AA, Haveman-Nies A, et al. Dietary Patterns, Cognitive Decline, and Dementia: A Systematic Review. Adv Nutr. 2015;6(2):154-168.

69. Gardener SL, Rainey-Smith SR, Barnes MB, et al. Dietary patterns and cognitive decline in an Australian study of ageing. Mol Psychiatry. 2015;20(7):860-866.

70. Dinan TG, Stilling RM, Stanton C, et al. Collective unconscious: How gut microbes shape human behavior. Journal of Psychiatric Research. 2015;63:1-9.

71. Franceschi C, Campisi J. Chronic Inflammation (Inflammaging) and Its Potential Contribution to Age-Associated Diseases. The Journals of Gerontology Series A: Biological Sciences and Medical Sciences. 2014;69(Suppl 1):S4-S9.

72. Forlenza OV, Diniz BS, Talib LL, et al. Increased Serum IL-1 $\beta$ Level in Alzheimer's Disease and Mild Cognitive Impairment. Dementia and Geriatric Cognitive Disorders. 2009;28(6):507-512.

73. Robertson DA, Savva GM, Kenny RA. Frailty and cognitive impairment-A review of the evidence and causal mechanisms. Ageing Research Reviews. 2013;12(4):840-851.

74. Trollor JN, Smith E, Agars E, et al. The association between systemic inflammation and cognitive performance in the elderly: the Sydney Memory and Ageing Study. Age. 2012;34(5):1295-1308. 